\title{
Kurzer Bericht über die 39. Versammlung deutscher Naturforscher und Aerzte.
}

$D_{\text {a }}$

der Plan unserer Zeitschrift nur ein kurzes Verweilen bei dem Gegenstande verstattet, so sei im Allgemeinen besonders hervorgehoben, dafs die Naturforscher auch diesmal ein ebenso uneigennütziges, als zum Theil wahrhaft aufopferndes Entgegenkommen fanden. Harmlos und freudig gab sich jeder Einzelne den vielfachen Anregungen einer anziehenden Geselligkeit in schöner Natur hin, nachdem er das Asyl verlassen, welches gemüthliche Gastfreundschaft an Stelle unheimlich theurer Gasthöfe ihm bereitet. Namentlich in kleineren Universitäts-Städten sind die Naturforscher-Versammlungen wahre Volksfeste, wenn man einmal den Kern der gebildeteren Bevölkerung unter Anschlufs der Uebrigen, und nicht die Uebrigen unter Ausschlufs der ersteren als Volk betrachtet. Im Gegensatz zu Stettin betheiligten sich in Giefsen bei den Festzügen und Parthien nach Schaumburg, Nauheim, Marburg, Ems auch die Damen lebhaft, wahrscheinlich weil diesmal ihr alter, beredter Verehrer, Professor Nöggerath nicht fehlte. Auf dem Festball liefs sich übrigens die Giessener männliche Jugend von den Naturforschern nicht leicht Concurrenz machen, und wird ihnen gewifs dankbar für denselben sein.

Nachdem die Versammlung durch den ersten Geschäftsführer Herrn Geh. Medicinalrath Werner eröffnet und eine Einladung zum Besuch des herrlich gelegenen Schlosses Schaumburg von Seiten Sr. K. K. Hoheit des Erzherzogs Stephan von Oesterreich mitgetheilt worden, waren die Zoologen so glücklich, sich um die Person des zweiten Geschäftsführers, Herrn Professor Leuckart, sammeln zu können, welcher als die Seele der ganzen Versammlung zu betrachten und von rastloser Thätigkeit war, dabei den Einzelnen fast bevorzugend, nicht vernachlässigend, practisch im Anordnen, ein Meister im freien Vortrag, gemüthlich, anregend, immer und überall auf dem Platze.

Nur in der Redaction des Tageblattes war die Geschäftsführung nicht ausreichend unterstützt. Der richtige Abdruck eines Namens in den Theilnehmerlisien war als eine Seitenheit zu betrachten und 
der Professor Schmidt mufste z. B. in der Ankündigung seines Vortrages über künstliche Schwämmezucht vom Setzer seine Schwämme in Schrveine verwandelt finden.

Tüchtige Zoologen waren neben den Entomologen stärker vertreleu, als in Stettin; von Letzteren waren aufser den weiter erwähnten anwesend: Professor Bilimek, Dickoré (Giessen), Professor Döbner, W. Fuchs (Berlin), Dr. Haag, Senator v. Heyden, OberLieutenant v. Heyden, Revisor Jännicke (Frankfurt a. M.), Prof. Kirsclbbaum, Prof. v. La Valette (Bonn), Riehl, S. Scheidel, Dr. Schneider, A. Schmidt (Frankfurt a. M.), Scidlitz (Dorpal), Pfarrer Scriba, Schulrath Suffrian.

Ueber die entomologischen Vorträge ist zu referiren:

Erste Sitzung. Präsident Prof. Troschel.

H. Rogenh ofer legł Brauer's Monographie über die Oestriden vor. Eiı von Prof. Schenck angekündigter Vortrag über die nassauischen Hummeln und ihre Schmarołzer fällt aus.

Zweite Sitzung. Präsident Prof. Kraufs.

Dr. Weif'smann trägt über Insectenenlwickelung vor. Redner zeigi, dal's zwei fundamental verschiedenc Arten der Metamorphose bei den Insecten vorkommen, deren eine nur wenigen Familien zukommt und durch die Musciden typisch repräsentirt wird, während für die andere die Tipuliden als Typus gelten können. Bei Letzteren bilden sich die Segmentanhänge von der Hypodermis der Larve aus, durch Ein- und Wiederausstülpung derselben. In die Ausstülpung kommt ein Tastnerv zu liegen, von dessen Neurilemma später die Entwickelung der inneren Theile des Anhangs (Muskeln, Sehnen, Tracheen) ausgeht. Wie in Entstehung der äufseren Theile, so unterscheidet sich die Metamorphose der Tipuliden auch in Um- und Neubildung der inneren Organe wesentlich von der der Musciden, und nicht minder durch die Lebenserscheinungen, welche das Puppenleben begleiten. Die Puppe der Musciden entbehrt der sichtbaren Lebensäufserungen, die der Tipuliden empfindet und bewegt sich wie die Larve. Die embryonale Entwickelung von Chironomus wird durch Wachspräparate, von F. Ziegler angefertigt, verdeutlicht.

Dritte Sitzung. Präsident Prof. Claus.

Ritter v. Frauenfeld trägt über die Metamorphose der Insecten vor und über zwei von der zoologisch.botanischen Gesellschaft herausgegebene Schriften. - v. Frauenfeld fand Auswuchs am Trifolium pratense, zog daraus Tychius polylineatus und Apion cavipes. Apion schmarotzl nur als Inquilin des durch Tychius ver- 
ursachten Auswuchses. - Im Stengel von Stachys liegt das Ei einer Blattwespe, welches vor der Embryonalentwickelung wächst. In Drontheim wurde auf einer Weide gleiches Wachsthum eines Eies einer Gallwespe gefunden. - An Chaerophyllum bulbosum wurde bei Eiern eines Rüsselkäfers, vielleicht Lixus, gleichfalls Wachsthum beobachtet. - Redner legt eine Copie von Hammerschmidt's Observationes physiologicae-pathologicae de plantarum gallarum ortu vor. - Das stärkere Auftrelen der Hessenfliege wurde heuer wieder beobachtet, es werden unter dem Namen verschiedene Fliegen zusammengeworfen.

Dr. Dohrn bemerkt dazwischen, dafs nach Loew Cecidomyia destructor und secalina zusammenfällt.

Ritter v. Frauenfeld machte die Bemerkung, dafs die Chloropiaceen saugen, die Cecidomyien nagen, letztere leben nahe der Wurzel der Pflanze, erstere höher. - Redner legt vor: Catalogus systemalicus Dipterorum Europae von Schiner.

Prof. Pagenstecher bemerkt, dafs bei der Cecidomyia ein Generationswechsel besteht. Unter Anguilluliden in Runkelrübenrückständen fand $\mathrm{Pagenstecher}$ Cecidomyia mit junger Brut im Innern; bestätigt daran die Beobachtung von Nic. Wagner; die Art ist neu; Redner betrachtet die Entstehung der Jungen und schildert den Verlauf der Entwickelung.

Prof. Cla us fragt, ob der erste Ort der Neubildung genauer bestimmt sei, erinnert an die von Dr. Weifsmann in voriger Sitzung gemachte Mittheilung.

Prof. Pagenstecher bemerkt, dafs zwei wenig bedeutende Häutungen an diesen Larven vorkommen, die er schildert; er bezeichnet die Fortpfianzung nicht als parthenogenetisch, sondern allgemein als ungeschlechtlich.

Prof. Keferstein schildert das Herz von Perophora und das Zustandekommen der wechselnden Contractionen.

Forstrath Hartig spricht über das Rückengefäls der Insecten, und erläutert dasselbe mit Zeichnungen. Das Rückengefäls ist an 3 Punkten aufgehängt; das wesentliche Organ der Saftaufnahme bei Dipteren und Hymenopteren-Larven liegt am Ende des Rückengefälses als dreilappiges Organ mit Spaltöffnungen an den Zipfeln, die geschlossen und eröffnet werden. Bei Ephemera- und Hemerobiuslarven findel eine Abweichung statt, wo die Aufnahme nicht am Zipfelapparate, sondern an der ersten Querfaltung stattfindet, ein Theil der Flüssigkeit strömt nach vorn, der andere nach hinten iu die Schwanzborsten. - Die Blutkügelchen stammen aus der Ge- 
websschicht unter dem Chitin; es werden kleine Stückchen aus dieser Zellschicht vorgetrieben, gelöst und gelangen in den Kreislauf, lagern sich am Aufnahmeapparate, und von dort werden dann einzelne Körner wie durch einen Kauapparat abgelöst. - Der Kreislauf soll diese Elemente in die Ausstülpung der neubildenden Anhänge treiben.

Prof. Claus bestätigt den Kreislauf in die Schwanzborslen und erwähnt von hier arterielle Gefälse.

Forstrath Hartig bemerkt, dafs die spindelförmigen Blutkügelchen erst bei Berührung mit Wasser sich abrunden; sie scheinen von einer Schleimhülle umgeben zu sein.

Forstrath Hartig spricht über die Parthenogenesis der Cynipiden. Die seit 25 Jahren angestellten Versuche, die Thiere aus Gallen zu ziehen, lieferten nur weibliche Thiere aus den Gattungen Cynips und Neuroterus; Männchen sollen danach absolut fehlen. Die Weibchen der unbemannten Arten legen gleich nach Vollendung der Entwickelung die Eier ab, die bemannten immer erst nach der Copula. - Redner beschreibt den Geschlechtsapparat der bemannten Gattungen, das Receptaculum enthält nach der Copula (bei Spathigaster) Spermatozoiden. Das Receptaculum findet sich auch bei den mannlosen Gattungen, aber stets ohne Samen. Redner giebt einen Ueberblick über die Entwickelung der Spermaelemente der Insecten.

Reallehrer Dickoré legt Zeichnungen von brasilischen Insecten vor.

Vierte Sitzung. Präsident Forstrath Hartig.

Im Anschlufs an seinen Vortrag vom vorhergehenden Tage schildert Herr Hartig die verschiedenen Spermaelemente genauer und geht sodann auf die Entwickelung der Insecten-Eier über.

Fünfte Sitzung. Präsident Prof. Pagenstecher.

Staatsrath v. Eichwald spricht über ein fossiles Insect. welches er für eine Ephemera-Larve hält. Es findet sich häufig in einem Kalkstein, den Redner für der Juraformation angehörig erklärt. Die Gattung wird Ephemeropsis genannt.

Prof. Leuckart spricht über die $Z_{\text {witter bei den Bienen, die }}$ zu Hunderten in einzelnen Stöcken vorkommen. Die Augen und Mundtheile sind mehr oder weniger drohnenartig, die Antennen bleiben denen der Arbeiterbienen ähnlich; die Hinterbeine verlieren vielfach die Eigenthümlichkeit der der Arbeiterbienen, der Stachelapparat geht allmählig verloren; bei einseitigem Verschwinden des Stachelapparates tritt ein Penis daneben auf. Die Erzeugung der 
Zwitter-Bienen durch die Königin ist erblich, soll sich jedoch in dem folgenden Geschlechte vermindern. Der Vortragende glaubl, dafs die Samenflüssigkeit in der Samentasche der Königin durch unvollständige Entwickelung der anhängenden Drüsen nicht vollständig befruchtungsfähig werde und erklärt dadurch das Auftreten der Zwitterbienen. -

Nachdem am 21. September 1864 in der Sitzung der zoologischen Section der 39. Versammlung deutscher Naturforscher und Aerzte ein Schreiben des Geh.-Rath Carus, als Präsident der Kaiserlichen Leopoldino-Carolinischen deutschen Academie, mitgetheilt worden, dals es erwünscht sein würde, wenn die Versammlung über ein allgemein für interessant erklärtes Thema einer neuen Frage sich aussprechen wolle, welches an Stelle der Preisaufgabe „die Naturgeschichte der Lampyris splendidula" treten möge, hatte die Section in der Sitzung vom 22. September zur Bestimmung eines solchen Themas eine Commission ernannt, bestehend aus den Herren Dohrn, Hartig, Leuckart, Pagenstecher, Troschel, welche sich für folgende Preisfrage erklärte:

Die Academie fordert:

Die Erläuterung des Verhältnisses zwischen geschlechtlicher und ungeschlechtlicher Forlpflanzung der Insecten durch Untersuchung der Generationsverhältnisse der Phytophthiren (Aphis, Coccus, Chermes).

Der Dank, welcher zum Schlufs dem ,um die ganze Versammlung, wie um die Section, so ausgezeichnet verdienten" Professor Leuckart ausgesprochen wurde, möge hier aus vollem Herzen wiederholt werden.

G. Kraatz. 


\section{$2 \mathrm{BHL}$ Biodiversity Heritage Library}

1864. "Kurzer Bericht über die 39. Versammlung deutscher Naturforscher und Aerzte." Berliner entomologische Zeitschrift / herausgegeben von dem Entomologischen Vereine in Berlin 8(3ロ4)402-406. https://doi.org/10.1002/mmnd.18640080324.

View This Item Online: $\underline{\text { https://www.biodiversitylibrary.org/item/36390 }}$ DOI: $\underline{\text { https://doi.org/10.1002/mmnd.18640080324 }}$

Permalink: https://www.biodiversitylibrary.org/partpdf/210051

\section{Holding Institution}

Smithsonian Libraries

\section{Sponsored by}

Smithsonian

\section{Copyright \& Reuse}

Copyright Status: Public domain. The BHL considers that this work is no longer under copyright protection.

This document was created from content at the Biodiversity Heritage Library, the world's largest open access digital library for biodiversity literature and archives. Visit BHL at https://www.biodiversitylibrary.org. 